\title{
Management of Post-intubation and/or Tracheotomy Tracheal Stenoses
}

\author{
P. Foccoli1, E. Scappaticci2, F. Rea33, F. Revello2, M. Bezzi1', S. Cavaliere1
}

Monaldi Arch Chest Dis 2011; 75: 1, 82-85.

Keywords: Trachea, Stenosis, Laser, Stent, Intubation.

1 U.O.C. di Endoscopia e Laserterapia dell'Apparato Respiratorio, A.O. Spedali Civili di Brescia,

2 Divisione di Pneumologia, A. Ospedaliero Universitaria, San Giovanni Battista, Torino,

3 Divisione e Cattedra di Chirurgia Toracica, Università degli Studi di Padova, Italy.

Correspondence: Dr. Pierfranco Foccoli, U.O.C. di Endoscopia e Laserterapia dell'Apparato Respiratorio, A.O. Spedali Civili di Brescia, P.zza Spedali Civili 1, 25123 Brescia, Italy; e-mail: pierfranco.foccoli@spedalicivili.brescia.it

The increasing number of intubations and percutaneous tracheotomies performed, together with the extended indication towards invasive mechanical ventilation, the prolonged time of survival and, as a consequence, increasing number of days spent in Intensive Care Units have determined an enlarged number of traumas of the upper airways at the bases of the increasing incidence of tracheal stenoses. About 2-3\% of patients who undergo intubations and/or tracheotomy develop tracheal stenosis [1] (Level of evidence: IIb).

\section{Aetiopathology}

The traumatic damage due to the decubitus of the endotracheal tube or tracheotomy cannula or caused by the pressure exerted by their cuffs are the most common causes of iatrogenic tracheal stenoses.

The ischemic and necrotic damage and possible cartilagineous involvement (fractures) lead to a redundant reparation process with consequent cicatritial tracheal stenosis [2,3] (Level of evidence: $I I b)$.

Several animal studies confirm that infections may also concur to the mucosal damage involved in the pathogenesis of airway stenoses [4] (Level of evidence: IIa).

\section{Diagnosis and classification}

Inspiratory or, less frequently, expiratory dyspnoea are the most frequent symptoms of respectively extra- and intrathoracic tracheal stenosis. Normally dyspnoea on effort is observed, with varying intensity depending on the calibre of the stenosis; when the tracheal diameter is less than 5 $\mathrm{mm}$ dyspnoea occurs also at rest. Cough is less frequently complained of, mostly with an accessional trend.

The first diagnostic element is represented by the clinical history (recent intubation) followed by pulmonary function tests with particular attention to the amputation of maximal flow rates.

A CT scan of the trachea may be very useful in order to obtain significant morphological data such as the site, calibre and extension of the stenosis, to describe the relationship with the adjacent anatomical structures and to reveal possible disruption of the tracheal wall. The gold standard in diagnosis is nonetheless represented by tracheoscopy since only the endoscopic evaluation can clearly define the type of stenosis (web-like, sleeve stenosis or granulomas), its extension, its exact location (distances from vocal cords and carina), the possible cartilaginous involvement with particular attention to the cricoids, the state of the mucosa (presence of flogosis, ulceration, necrosis or fibrosis), the presence of tracheal malacia and the exact calibre. Moreover tracheoscopy allows for a specimen for histological definition and microbiological studies aimed to reveal infections.

On the basis of the endoscopical characteristics several types of stenosis can be distinguished:

- Granulomas (at the tip of the cannula, on the stoma site, etc.)

- Fibrotic bridges

- "Pseudoglottic" stenosis ("A" shaped stenosis due to fracture of a cartilaginous ring secondary to tracheotomy)

- True cicatricial stenosis, further distinguishable into

- Web-like stenosis mostly circumferential strictures of the trachea involving the mucosa of a short segment (maximum $1 \mathrm{~cm}$ in length)

- Complex stenosis sleeve strictures of the trachea longer than $1 \mathrm{~cm}$ and often associated with various degrees of cartilage involvement, malacia and flogosis.

\section{Therapy}

It is very difficult to stratify patients for degree of illness and co-morbidities, which in turn makes 
it difficult to carry out large and randomised therapeutical trials in order to determine the best therapeutic strategy.

Patients frequently refer to the emergency and it is mandatory to treat them immediately, making it impossible to organise stratified studies.

In literature there are only a few clinical reports, retrospective analysis or, in few cases, notcontrolled and not-randomised perspective studies. According to the latter, the therapeutic approach to cicatricial tracheal stenosis has to be multidisciplinary, concerning the otolaryngologists and/or thoracic surgeons and interventional pneumologists.

The indications towards endoscopic treatment of cicatricial tracheal stenosis are still much debated, since in this field surgery has made remarkable progresses with reduction of mortality and morbidity and with improvement of quality and stability of outcomes [5] (Level of evidence: IV).

According to many authors surgical resection with end-to-end anastomosis represents the elective choice with over 90\% success rate [6-8] (Level of evidence: III). Therapeutic bronchoscopy is considered only a "pro-tempore" treatment before surgery, particularly in critical situations, aimed at avoiding more invasive procedures such as tracheotomy $[5,7,9]$ (Level of evidence: III).

However in some cases surgery is contra-indicated due to the length of the stenosis or severe associated pathologies; in these situations less invasive endoscopic treatments are taken into consideration.

This therapeutic options include mechanical dilation, methods of local tissue ablation, possibly followed by endoprosthesis placement; all these procedures are performed through the rigid bronchoscope, under general anaesthesia [10-12] (Level of evidence: $I I b$ ).

Rigid bronchoscopy offers several advantages:

- It supports the airways' patency allowing ventilation and oxygenation

- It offers a better vision

- It permits the introduction of various tools at the same time (dilators, forceps, rigid suction tubes, optics)

- It permits stent placement

- It permits to front complications [11, 13, 14] (Level of evidence: III).

\section{Endoscopic therapeutic techniques}

\section{Mechanical dilation}

It consists of enlarging the tracheo-bronchial lumen with the rigid technique under general anaesthesic. It is also possible to use various types of dilators (probes with balloon, Jackson's dilators of progressive calibre) in local anaesthesia, conscious sedation or general anaesthesia.

Most authors perform mechanical dilation with rigid technique, using tubes of increasing calibre, because of the advantages associated to the good management of airways and the low incidence of complications [11, 13-16] (Level of evidence: III).

\section{Electrocauterication (EC), Argon-Plasma} Coagulation (APC) and Criotherapy (Cryo)

These are thermal ablation modalities consisting in the application of high frequency electric current (EC and APC) or very low temperature $\left(-40^{\circ} \mathrm{C}--60^{\circ} \mathrm{C}\right)(\mathrm{Cryo})$; they can be endoscopically performed in rigid or flexible bronchoscopy, in general or local anaesthesia respectively.

Most publications on this subject concern the treatment of malignant lesions. If used in the treatment of post-intubation tracheal stenosis EC and APC can be fibro-genetic and Cryo is ineffective because of the lack of blood vessels; moreover it can be dangerous for the possible persistence of iced tissue in the airways. These methods, especially APC, may only be used in granulomas [1719] (Level of evidence: III).

\section{Laser (Light Amplification by Stimulated Emission of Radiation)}

An external energy source, usually optical or electrical, is used to stimulate an active substance (normally Nd-YAG or Diodes) container within the core of the laser.

For the treatment of cicatricial stenoses the most suitable technique is based on radial laser incisions followed by gentle balloon (less traumatic) or tube dilation (visual control). Contact laser is preferably used, with high absorption coefficients and low scattering coefficients in order to localize its effect on the cut surface avoiding excessive heating of the surrounding tissues with consequent fibrosis. The laser beam is interrupt when the incision is visibly sufficient, without redundant energy delivery $[10,11,20]$.

\section{Stents}

Tracheo-bronchial prostheses are normally silicon cylindrical stents, metallic ones or combined (silicon+metallic). They support the airway walls maintaining the calibre obtained thanks to laser assisted endoscopic dilation.

Stenting is indicated in case of unstable stenoses, quite likely to recur

It is normally a temporary item aimed at defending the airway calibre as long as the fibro-cicatricial process comes to a head; therefore prostheses must be easily removable which is true for silicon stents, but not for metallic or combined ones. As a consequence, at present, the most suitable are silicon stents.

Vocal cord paralysis represents relative contraindications to stent placement since it compromises cough efficacy increasing the risk of sputum retention [21, 22] (Level of evidence: III).

Possible/Infrequent complications are dislocation, granulomas, obstruction by sticky secretions or bacterial over infections with consequent "foetor ex ore".

\section{Medical Therapy}

The utility of antibiotic therapy after interventional bronchoscopy is not proved except that in 
bacterial endocarditis. Nevertheless it is probable that, when the treatment has been particularly traumatic, the antibiotic treatment could be useful in preventing over infection which could contribute to ristenosis. There is not any evidence of the role of steroids in prevention of recurrence, but also in this case it is probable that anti-inflammatory therapy, reducing flogosis, could contribute towards the stabilisation of the airway damage. One experimental study on dogs demonstrates the role of antibiotic and steroid therapy in protecting the tracheal wall to stenosis after a trauma [23] (Level of evidence: IIa).

It has been hypothesised that mitomycin (an antiblastic drug inhibiting fibroblastic proliferation) could have a role in preventing stenosis recurrence. Randomized animal studies offer contrasting results $[24,25]$ (Level of evidence: IIa); several non controlled clinical studies on human seem to prove its efficacy in preventing recurrence of stenosis [26, 27] (Level of evidence: IIb).

\section{Surgical resection}

Surgical resection with end-to-end anastomosis is widely considered as the elective treatment [6-8] (Level of evidence: III).

Preoperative bronchoscopy is essential to plan surgery that can be absolutely or temporary not indicated. Absolute contraindications are severe and irreversible co-morbidities and too long stenosis (more than $50 \%$ of tracheal length).

In all temporary contraindication to open surgery (acute flogosis, infections or not yet well defined stenosis...) and in case of critical stenoses (diameter $<5 \mathrm{~mm}$ ) the endoscopic treatment is advisable, even repeatedly, according to the most experienced surgeons; in fact, the optimal surgical timing increases the results and decrease the complications [5-8, 22, 28] (Level of evidence: III).

\section{Complications}

Fibrine engorgement is a frequent complication of endoscopic laser treatments, whereas airway wall perforation occurs anecdotically. In case of stent placement possible complications are dislocation, granulomas, obstruction by sticky secretions or bacterial over infections with consequent "foetor ex ore". Major or permanent complications are never described [10, 22] (Level of evidence: III).

Minor complications of open surgery such as vocal cord paralysis [5], have largely reduced ranging from 5 to $15 \%$, with a very low mortality rate, although some cases of death are described even in the best series [6-8, 29] (Level of evidence: III).

\section{Follow-up}

Asymptomatic patients should not undergo untimely/early bronchoscopic evaluations since it is largely accepted that if recurrences occur it normally happens 20-40 days after the endoscopic treatment.
Even in case of stent placement a planned endoscopic follow up in absence of renewal of symptoms can not be justified since it does not reduces the number of complications. On the other hand an endoscopic evaluation at one year from treatment may be very useful to evaluate the final results [30] (Level of evidence: III).

\section{Results}

All studies on this topic report different success rates of the endoscopic therapy ranging from 32 to $66 \%$ depending on the type of stenosis and the endoscopic technique applied.

For the treatment of simple lesions, namely granulomas and web-like stenoses, the success rate is almost $100 \%[10,20,31]$ (Level of evidence: III).

\section{Recommendations}

- The therapeutic approach towards tracheal stenoses must involve otolaryngologists and thoracic surgeons as well as interventional pneumologists. The endoscopic treatment is the only treatment available for those patients not suitable for surgery because of the excessive length of the stenosis or because of the presence of severe co- morbidities (Grade B).

- The urgent endoscopic treatment of critical web-like or complex stenoses (diameter $<5 \mathbf{~ m m}$ ) is universally accepted since it permits to overcome the acute phase without performing more invasive and dangerous procedures, namely tracheotomy (Grade B).

- When one or more cartilaginous rings are involved the endoscopic treatment is normally contraindicated unless surgery is not feasible (Grade B).

- The endoscopic treatment is the gold standard for simple lesions, namely granulomas, fibrotic bridges or weblike stenoses, with a very high success rate (Grade B).

- In cases of potentially complex surgical tracheal stenoses the choice of treatment is quite debated. In all temporary contraindications to open surgery (acute flogosis, infections or unstable stenoses) the endoscopic treatment allows a variable period of palliation of symptoms minimising the use of steroid drugs which could risk the surgical anastomosis. In all cases of transient contraindications to surgery the endoscopic treatment, even repeated, allows an optimal surgical timing with better surgical results and fewer complications (Grade B). 
- In $66 \%$ of complex stenoses Laser Assisted Mechanical Dilation (LAMD) with or without stenting can be efficacious with very few transient complications and no mortality. Nonetheless the surgical resection and anastomosis also achieves definitive results. The choice between these two kinds of treatments is quite debated, often influenced by the clinical and psycho-neurological conditions of the patients (Grade B).

\section{References}

1. Stauffer JL, Olson DE, Petty TL. Complications and consequences of tracheal intubation and tracheotomy. A prospective study of 150 critically ill adult patients. Am J Med 1981; 70: 65-76.

2. Cooper JD, Grillo HC. Experimental production and prevention of injury due to cuffed tracheal tubes. Surg Gynecol Obstet 1969; 129: 1235-41.

3. Knowlson GTG, Bassett HFM. The pressure exerted on the trachea by tracheal inflatable cuffs. Br J Anaesth 1970; 42: 834-7.

4. Squire R, Brodsky 1, Rossman J. The role of infection in the pathogenesis of acquired tracheal stenosis. Laryngoscope 1990; 100: 765-70.

5. Wain JC Post-intubation tracheal stenosis. Chest Surg Clin N Am 2003; 13: 231-246.

6. Couraud L, Jougon JB, Velly JF. Surgical treatment of nontumoral stenoses of the upper airway. Ann Thorac Surg 1995; 60: 250-9.

7. Grillo HC, Donahue DM, Mathisen DJ. Postintubation tracheal stenosis. Treatment and results. J Thorac Cardiovasc Surg 1995; 109: 486-93.

8. Rea F, Callegaro D, Sartori F. Benign tracheal and laryngotracheal stenosis: surgical treatment and results. Eur J Cardiothorac Surg 2002; 22: 352-6.

9. Grillo HC. Urgent treatment of tracheal obstruction. In: Grillo HC (Ed.) Surgery of trachea and bronchi. BC Decker Inc., 2004

10. Brichet A, Verkindre C, Dupont J, Marquette CH. Multidisciplinary approach to management of postintubation tracheal stenoses. Eur Resp J 1999; 13: 888-893.

11. Cavaliere S, Foccoli P, Toninelli C. Endobronchial laser treatment. Eur Respir Monograph 1998; 3: 49-64.

12. Cavaliere S, Bezzi M, Toninelli C, Foccoli P. Management of post-intubation tracheal stenoses using the endoscopic approach. Follow-up of 73 consecutive patients over a four-year period. Monaldi Arch Chest Dis 2007; 67: 73-80.

13. Cavaliere S, Foccoli PF, Toninelli C. Trattamento laser endobronchiale Eur Resp Monograph Ed. Italiana, vol 5, Monografia 3, dic 2001, pp 48-61.

14. Cavaliere S, Dumon JF. Laser Bronchoscopy from Bolliger CT, Mathur PN (eds): Interventional Bronchoscopy. Prog Respir Res. Basel, Karger, 2000, vol 30, pp 108-119.

15. Lee $\mathrm{KH}$, Ko GY, et al. Benign tracheobronchia stenoses: long-term clinical experience with balloon dilation. J Vasc Intervent Radiol 2002; 13: 909-914.

16. Mayse ML, Greenheck J, et al. Successful bronchoscopic balloon dilation of non-malignant tracheobronchial obstruction without fluoroscopy. Chest 2004; 126: 634-637.

17. Tremblay A, Marquette CH. Endobronchial electrocautery and argon plasma coagulation: a practical approach. Can Respir J 2004; 11: 305-10.

18. Grund KE, StoreK D, Farin G. Endoscopic argon plasma coagulation (APC) first clinical experiences in flexible endoscopy. Endosc Surg Allied Technol 1994; 2: 42-6.

19. Maiwand MO, Zehr KJ, Dyke CM, et al. The role of cryotherapy for airway complications after lung and heart-lung transplantation. Eur J Cardiothorac Surg 1997; 12: 549-54.

20. Shapshay SM, Beamis JF, Hybels RL, Endoscopic treatment of subglottic and tracheal stenosis by radial laser incision and dilatation. Ann Otol Rhinol Laryngol 1987; 96: 661-664.

21. Colt HG The Art and Science of Airway Stenting. $J$ Bronchol 1995; 2: 263-266.

22. Puma F, Ragusa M, Avenia N, The role of silicon stents in the treatment of cicatricial tracheal stenosis. J Thorac Cardiovasc Surg 2000; 120: 1064-9.

23. Croft CB, Zub K, et al. Therapy of iatrogenic subglottic stenosis: a steroid/antibiotic regimen. Laryngoscope 1979; 89: 482-9.

24. Eliashar R, Eliashar I, et al. Can topical mitomycin prevent laryngotracheal stenosis? Laryngoscope 1999; 109: 1594-600.

25. Correa AJ, Reinisch L, et al. Inhibition of subglottic stenosis with mitomycin-C in the canine model. Ann Otol Rhinol Laryngol 1999; 108: 1053-60.

26. Rahbar R, Shapshay SM. Mitomycin: efficacy on laryngeal and tracheal stenosis, benefits and complications. Ann Otol Rhinol Laryngol 2001; 110: 1-6.

27. Perepelitsyn I, Shapshay SM. Endoscopic treatment of laryngeal and tracheal stenosis- has mitomycin-C improved the outcome? Otolaryngol Head Neck Surg 2004; 131: 16-20

28. Grillo HC. Urgent treatment of tracheal obstruction. In Grillo HC (Ed.) "Surgery of trachea and bronchi" BC Decker Inc., 2004.

29. Har-El G, Lim JW, Wynn R. Tracheal resection with end-to-end anastomosis for benign tracheal stenosis. Ann Otol Rhinol Laryngol 2004; 113: 613-17.

30. Matsuo T, Colt HG. Evidence against routine scheduling of surveillance bronchoscopy after stent insertion. Chest 2000; 118: 1455-59.

31. Reza Nouraei SA, Khalid G, Patel A, et al. Outcome of endoscopic treatment of adult postintubation tracheal stenosis. Laryngoscope 2007; 117: 1073-1079. 